\title{
An Energy-efficient Point Coordination Function Using Bidirectional Transmissions of Fixed Duration for Infrastructure IEEE 802.11 WLANs
}

\author{
Raúl Palacios, Fabrizio Granelli \\ University of Trento \\ Trento, Italy \\ \{palaciostrujillo,granelli\}@ disi.unitn.it
}

\author{
Danica Gajic, Christian Liß \\ InnoRoute $\mathrm{GmbH}$ \\ Munich, Germany \\ $\{$ gajic,liss\}@innoroute.de
}

\author{
Dzmitry Kliazovich \\ University of Luxembourg \\ Luxembourg \\ \{dzmitry.kliazovich\}@uni.lu
}

\begin{abstract}
The Point Coordination Function (PCF) of the IEEE 802.11 standard represents a well-known Medium Access Control (MAC) protocol providing Quality-of Service guarantees in Wireless Local Area Networks (WLANs). However, with the currently employed polling mechanism WLANs consume a significant amount of the energy resources from batterypowered user devices. To provide energy saving, an improved MAC protocol is presented in this paper, where bidirectional transmissions of fixed duration are incorporated into PCF in order to enable dynamic scheduling of real-time traffic. Based on this new strategy, wireless access points (APs) can estimate the proper duration of the Contention Free Period (CFP), in order to allow mobile stations to acknowledge any received data packet with a data packet equal to the received packet in size. Having this information, a mobile station, following the data exchange with the AP, can determine its wake-up timer and activate the sleep mode for the rest of the CFP interval. Comprehensive computerbased simulations demonstrate the feasibility of the proposed MAC improvements to achieve energy efficiency with negligible impact on packet delivery delay.
\end{abstract}

\section{INTRODUCTION}

With the proliferation of portable devices equipped with Wireless Local Area Network (WLAN) adapters, the IEEE 802.11 technology [1] has become a very popular solution to provide fixed and mobile users with short-range wireless connectivity. However, the high energy consumption of WLAN interfaces significantly constrain the energy resources of mobile devices, which are typically powered by small batteries [2], [3]. Thus, prolonging the battery life for mobile devices is an urgent demand from mobile users in order to enjoy seamless mobile services [4].

A major cause of energy consumption in an infrastructure WLAN is the Access Point (AP) coordinating an access to the shared channel between mobile stations located in the coverage area, Fig. 1. The IEEE 802.11 standard defines two medium access control (MAC) protocols: the Point Coordination Function (PCF) and the Distributed Coordination Function (DCF). The AP specifies through beacons the access mode that stations have to follow to transmit or receive data, based on superframes that are divided into contention free periods

*This work is funded by the GREENET research project (PITN-GA-2010264759).

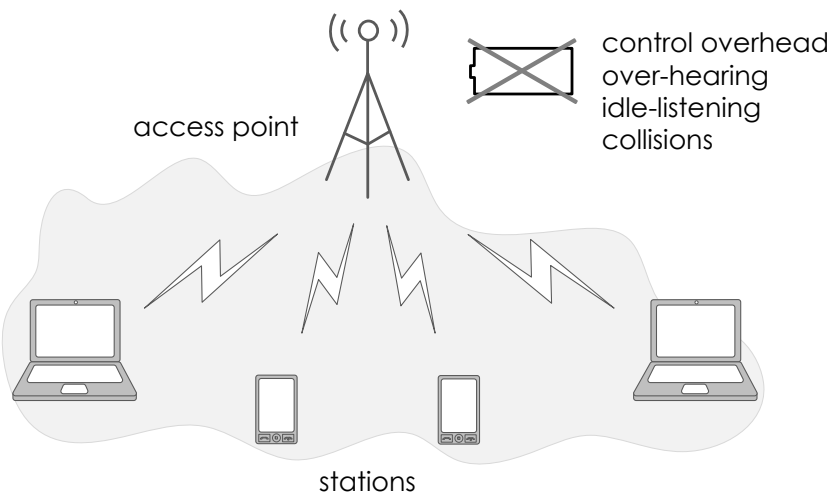

Fig. 1. An infrastructure wireless local area network.

(CFPs) for the PCF access and contention periods (CPs) for the DCF access. To access the channel, employing either PCF or DCF, WLAN stations need to continuously monitor the channel activity, thus consuming a significant amount of energy resources to receive packets intended for other stations.

Since DCF is the fundamental mechanism for channel access in the IEEE 802.11 standard, extensive research has been undertaken on improving its performance, including energy efficiency [5]. On the other hand, PCF is optional and has received less attention in the literature, despite its capability to improve performance under heavy traffic loads [6] and enable Quality of Service (QoS). However, the polling strategy employed in PCF leads to non-optimal performance in terms of energy consumption and packet transmission delays [7]. As the fundamental characteristics of PCF have survived across the new amendments of the standard, e.g. the Hybrid Controlled Channel Access (HCCA) defined in IEEE 802.11e, significant studies need to be undertaken to improve performance and energy efficiency in infrastructure WLANs.

This paper presents a modified version of the PCF function, called Bi-Directional PCF (BD-PCF), aiming at minimization of energy consumption at the mobile nodes of a cell. In particular, an energy-saving strategy is incorporated into standard PCF, where each station, after being served by the AP, can fall 
asleep for the rest of the CFP interval and wake up for the CP period. While asleep, most of the hardware components of the WLAN interface are turned off, hence significantly reducing energy consumption. The proposed approach is based on our previous findings presented in [8].

The main contributions of this work are summarized as follows:

1) Design of IEEE 802.11 MAC protocol enhancements.

2) Performance evaluation of BD-PCF compared with PCF in extensive simulations.

3) Presentation of simulation results showing energy consumption, throughput and delay, with multiple stations and heavy traffic loads.

The remainder of the paper is organized as follows. Section II provides an overview of the related work and states the motivations of this work. The MAC improvements proposed for PCF are then introduced in Section III. Section IV describes the performance evaluation of BD-PCF with respect to the standard. Conclusions are drawn in Section V.

\section{RElated WORK AND Motivations}

The IEEE 802.11 standard defines two modes of Power Management (PM): the active mode and the Power Save (PS) mode. Stations operating in the active mode are awake and listen to the WLAN channel. Their transceivers are fully powered to transmit and receive frames. On the other hand, mobile stations in PS mode save power by turning off their transceivers and wake up only to listen to selected beacons.

In infrastructure-based networks, all stations, either in active or PS mode, can access the WLAN channel during either the CFP or CP period. During CFP, the AP operates as a point coordinator ( $\mathrm{PC})$, employing the polling strategy to individually serve the stations located in the polling list. Since PCF is an optional coordination function, and not implemented in all standard-compliant devices [7], $\mathrm{CP}$ is necessary to guarantee the access to the channel for DCF stations.

In the legacy IEEE 802.11 PCF protocol, the AP maintains a polling list and determines which station has the right to transmit a frame. PCF was originally designed for QoS provisioning over WLANs. However, the polling mechanism used in PCF presents several limitations in terms of delay and energy consumption [7].

1) Protocol overhead. During the polling process, the AP can send poll messages, data packets and acknowledgments (ACKs) to stations in the polling list. Polled stations have to acknowledge the polls and the data packets with ACK and a data packet, or a null packet (if no data). Thus polls, nulls and ACKs represent a source of bandwidth and energy waste. To reduce the overhead associated to the polling process, a modified operation of PCF was proposed in [9], where a CFP interval is divided into the distributed polling protocol period for uplink transmissions, without any polling overhead, and the real-time traffic downlink period. In [10], [11] a reduction in the number of poll packets is achieved by detecting periods of inactivity of polled stations.
2) Packet transmission duration. The transmission time of a polled station is unknown during CFP. Any station that is polled by the AP can send a single frame of any length of up to 2312 bytes. This may result in unfairness. To solve this problem, the SuperPoll protocol was proposed in [12], where the list of stations with the right to transmit in a certain period is provided within a superpoll frame at the beginning of a CFP interval. This approach implies that the length of data frames of polled stations must be fixed in CFP.

3) Monitoring the channel activity to transmit packets. During the whole duration of CFP, all stations have to constantly listen to the WLAN channel, thus consuming a significant amount of energy to receive packets intended for other stations. When the number of active stations is large, the last station must consume energy to overhear all the transmissions of all stations to and from the AP. To optimize energy consumption, a group-polling frame with the polling order of stations and their assigned transmission times was introduced in [13], where a station is in the doze state most of CFP, except for when it intends to transmit a data frame. In [7] the Unified Point Coordination Function (UPCF) was proposed, which defines a vector-list poll frame and a power-conserving scheduling so that PS stations can spend as less energy as possible during the polling activity. On the other hand, Ref. [14] presented an energy-efficient multi-polling mechanism with a PM strategy based on optimal wake-up intervals that comply with a desirable bandwidth utilization.

In our previous work [8], MAC enhancements for PCF were proposed to a) reduce the overhead associated to the polling process and b) solve the problem of unpredictable medium occupancy of polled stations. In particular, MAC modifications were introduced to allow mobile stations to acknowledge any received data packet with a data packet equal to the received packet in size. Thus, data packets are established in accordance with received data packets and less overhead is required during the polling process.

In this paper, the approach discussed in [8] is extended to provide an energy-saving strategy that allows polled stations to return to the doze state, after exchanging data with the AP. Therefore, the energy consumed by admitted stations to monitor the channel activity can be significantly reduced. Unlike other approaches described in this section, our proposal is characterized by a low protocol overhead, dynamic scheduling of real-time traffic flows, energy efficiency and feasibility of implementation and standardization.

\section{Protocol Design}

This section provides a detailed description of the required modifications for PCF, by first giving an overview of the standard PCF operation.

\section{A. IEEE 802.11 PCF overview}

The PCF mode is a centrally-controlled mechanism that relies on the polling access and provides connection-oriented services with a certain level of QoS. 


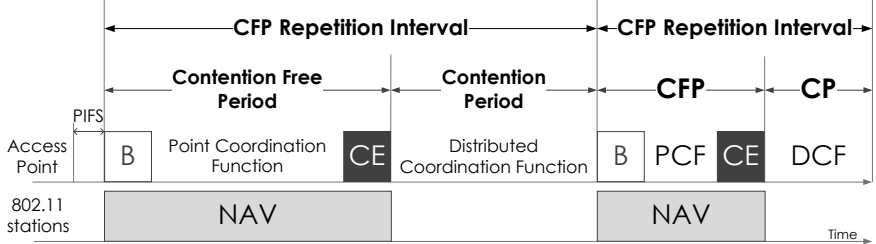

Fig. 2. IEEE 802.11 PCF uses a CFP repetition interval with CFPs and CPs.

When PCF operates in an infrastructure WLAN, the AP announces a CFP repetition interval in which CFP is followed by $\mathrm{CP}$, as illustrated in Fig. 2. During CFP all stations have to access the WLAN channel, using PCF. After that, the AP and the stations enter a CP interval, where the DCF is executed.

CFP begins with a beacon (B) that is periodically transmitted following the PCF Inter Frame Space (PIFS). With a beacon the AP can take control of the WLAN channel after CP. The beacon frame contains information related to the duration of both CFP and CP, specified with the CFP repetition interval and the maximum allowable duration of CFP (CFP-Max-Duration). The latter is used to update network allocation vectors (NAVs) at the mobile stations. Both the CFP repetition interval and the maximum allowable duration can be dynamically adjusted by the AP according to the data traffic requirements. The value of CFP-Max-Duration should be selected to allow at least one data packet transmission during $\mathrm{CP}$, as required for the coexistence of contention and contention-free traffic. CFP ends with a CFP End (CE) control packet transmitted by the AP, which also signals the beginning of a CP period. CP periods are used by the stations for the transmission of data packets or re-association control packets to the AP to join or leave the polling list.

Fig. 3 provides an example of a PCF access and corresponding energy consumption during transmission, reception and idle periods. The figure is composed of three parts. On the top, the polling activity and the energy consumption of the AP are shown. In the middle, channel access and the energy consumption of polled stations are depicted. In the bottom, the MAC activities and the energy consumption of other stations are displayed. In this example, a CFP interval is initiated with a B frame After SIFS, the AP combines a poll packet with a data packet (D1), sending it to Station 1. After receiving the combined packet, Station 1 acknowledges the poll and the data with a combined packet containing an ACK and data packet (U1) to the AP. Then, the AP sends an acknowledgment to Station 1, combined with a poll packet with a data packet (D2) directed to Station 2. Due to channel errors or a collision, there is a missing response from Station 2 during a PIFS interval. Thus, the AP polls Station 3 with a polled packet combined with a data packet (D3). Station 3 has no data to transmit and replies an ACK with a null control packet to the AP. The CFP interval ends with the transmission of the CE packet.

\section{B. MAC enhancements for PCF}

The proposed PCF function extends standard IEEE 802.11 PCF with an improved version of bidirectional transmissions

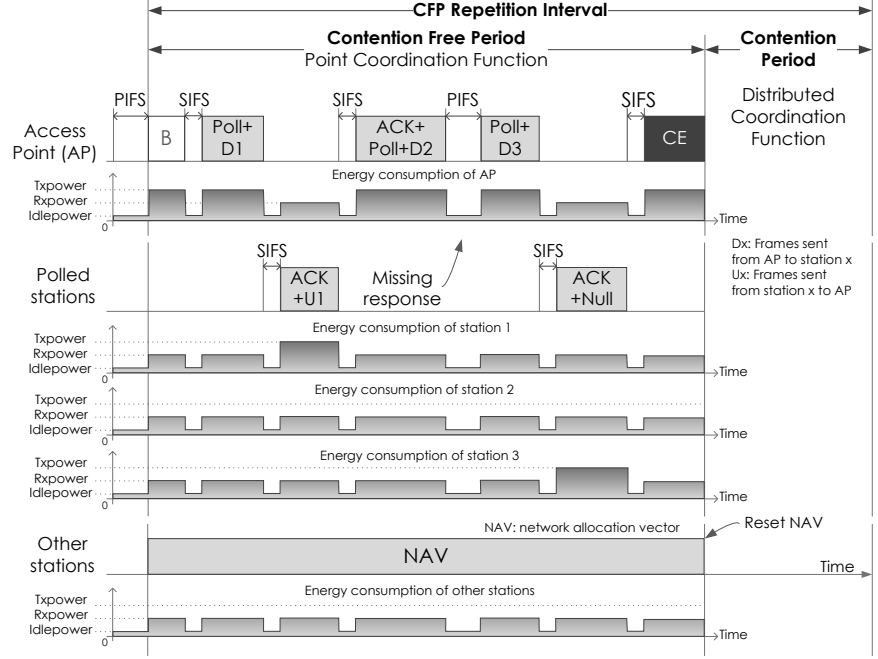

Fig. 3. Operation and energy consumption of IEEE 802.11 PCF.

[8] and a low-complexity mechanism that allows admitted stations to save energy during the polling process. By following these strategies, the MAC modifications for PCF are described as follows.

With the introduction of bidirectional transmissions, the access to the WLAN channel for a station in the polling list is granted one SIFS interval after receiving the data packet from the AP. The polled station can then acknowledge the reception of the data packet, by sending a data packet of equal duration of the downlink data packet to the AP. If the polled station has no data to transmit, it only acknowledges (ACK) the data packet, or replies with a null packet whether the AP sent a poll packet because of no data for the station. Therefore, the polling overhead can be minimized when the AP has downlink data for the stations in the polling list, thus improving channel utilization.

Since the duration of uplink transmissions is fixed by the duration of downlink transmissions, the transmission time of stations in the polling list can be known in advance, from the data packets buffered for these stations at the AP. Thus, the AP can properly determine the duration of a CFP interval to allow an uplink transmission for each station in the polling list. However, not all the stations in the polling list might have a data packet to transmit. The stations intending to leave the polling list because of no more data to send might fail to reassociate with CP. Since DCF is a contention-based protocol, the stations have to compete with all other stations in the same cell for channel access, and might need several CPs to send the reassocation frames. This aspect might degrade the performance of modified PCF incorporating bidirectional transmissions. As a solution to this issue, one bit can be taken from the MAC header of the data frame used in CFP, to inform about having more data to transmit. Once an admitted station has no more data to send, it should set the more data bit to 0 in the MAC header of the last data packet, as proposed in [7]. With this information, the AP will remove that station 
from the polling list, in an easy and quick manner, without performing a reassociation in $\mathrm{CP}$.

When the AP has no data to transmit to a given station in the polling list, it sends a poll packet to which the station can reply with a data packet of an arbitrary length (up to a maximum of 2312 bytes). This might have a negative effect on the performance of other stations in the polling list. To solve this limitation, the AP can maintain the information related to the duration of the last downlink data packet sent to each station in the polling list, with a record. Likewise, each station can have knowledge of the duration of the last uplink data packet transmitted to the AP. When a station receives a poll packet without a data packet, it will send an uplink data packet of equal duration of the last uplink data packet. Hence, the AP can estimate a proper duration of a CFP interval, based on own information on downlink packets and the polling list.

Some underperformance of modified PCF with bidirectional transmissions might also occur if a station has an uplink packet of shorter duration of the downlink packet, since the duration of the uplink packet must be fixed by the downlink packet to determine a proper CFP duration. This issue can be faced by adding a sequence of zero bits (fill bits) at the end of the uplink packet to match the size of the downlink packet. Although the channel utilization can be minimally affected with the fill bits, all the previous modifications of PCF can provide a precise estimation of the next CFP, which can be used by the AP to enable energy saving for admitted stations in the polling list.

After estimating the CFP interval, the AP updates the CFPMax-Duration field. All stations update their NAVs with CFPMax-Duration when the beacon is transmitted. During the polling process, each pollable station then monitors the time elapsed from the transmission of the beacon until receiving the ACK of its uplink data packet and immediately after returns to the doze state until the end of the CFP interval. With the CFP-Max-Duration and the time elapsed to receive ACK, the station can calculate the sleep period and set its wake-up timer to the beginning of $\mathrm{CP}$.

In the presence of channel errors or collisions, the AP will wait for PIFS and send a data packet to the next station in the polling list. Under this scenario, the stations with bad channel conditions might stay awake for the entire CFP, hence consuming extra energy. In addition, the duration of the CFP interval will be changed. Thus, PS stations will remain longer in the doze state, and awake when CP is already initiated. To prevent potential instability issues, the AP will not announce a new CFP interval until the CFP-Max-Duration has expired, thus allowing PS station to wake up and follow the next CFP repetition interval. In addition, station-to-station communications (via the AP) involving PS stations will be frozen during that $\mathrm{CP}$ interval, and the data packets will be buffered at the AP until CFP-Max-Duration expires.

The operation and energy consumption of PCF modified with the MAC enhancements aforementioned are summarized in Fig 4. From the figure, it is worth noting that the last station in the polling list suffers from high energy consumption when the number of stations is large. Since PCF uses the round-robin

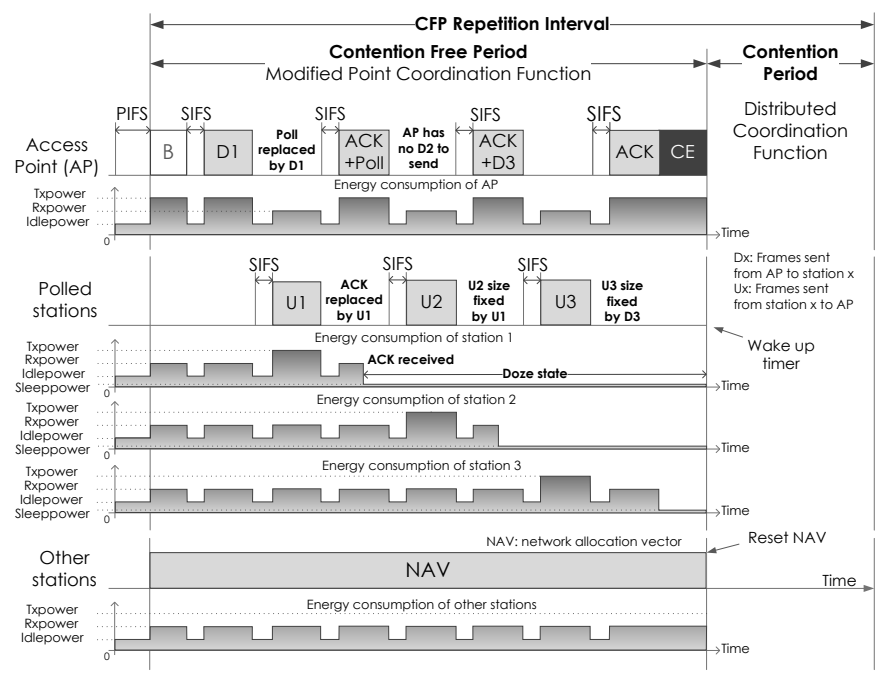

Fig. 4. Operation and energy consumption of BD-PCF.

(RR) scheme to schedule the polling order, the list of stations is established with the association IDs (AIDs) of admitted stations in ascending order. To reduce the energy consumption of the last station, a cyclic RR algorithm is incorporated into BD-PCF, where the order of stations will be constantly moving from the first position to the end of the polling list in each CFP interval.

Indeed, these modifications can help improve the energy efficiency of the 802.11 PCF protocol, with minimum impact on channel utilization and packet transmission delays. However, the performance of the proposed PCF MAC protocol should be carefully analyzed in the presence of channel errors, and provide the required improvements. This aspect will be part of the future work on this topic.

\section{Performance Evaluation}

This section analyzes the performance of BD-PCF against PCF, with a description of the simulation model followed by a discussion on the simulation results.

\section{A. Simulation Setup}

A custom-designed simulator in Python was developed to verify the performance of the proposed BD-PCF in comparison with the standard PCF. The simulation scenario considers a single WLAN cell with an AP and a number of associated stations. All stations are within the transmission range of each other. The simulation model assumes a time distribution of consecutive CFPs interleaved by PIFS for the duration of 100 beacons. There are no CPs included to properly evaluate the MAC modifications against standard PCF. At the beginning of each CFP interval, all stations, including the AP, generate fixed-size data packets. The AP polls and sends data to all the associated stations during the entire CFP. For every transmitted data packet the stations are granted the opportunity to send a data packet back to the AP. Focusing on the MAC layer, the channel model assumes ideal channel conditions, hence making the obtained results to correspond to an upper-bound 
TABLE I

SIMULATION PARAMETERS.

\begin{tabular}{c|c|c|c}
\hline Parameter & Value & Parameter & Value \\
\hline SIFS & $10 \mu \mathrm{s}$ & Preamble & $16 \mu \mathrm{s}$ \\
DIFS & $28 \mu \mathrm{s}$ & Signal & $4 \mu \mathrm{s}$ \\
PIFS & $19 \mu \mathrm{s}$ & Service & 6 bits \\
Tail & 16 bits & MAC header & 34 bytes \\
Beacon/Poll/CF-end & 20 bytes & ACK & 14 bytes \\
Data packet (MPDU) & 1500 bytes & Payload & 1466 bytes \\
Data rate & 48 Mbps & Control rate & 6 Mbps \\
Transmit state & 1.65 Watts & Receive state & 1.4 Watts \\
Idle state & 1.15 Watts & Doze state & 0.045 Watts \\
No. of sta. & $2-50$ & No. of beacons & 100 \\
\hline
\end{tabular}

performance of BD-PCF. Table I summarizes the simulation parameters, which follow the IEEE 802.11g MAC/PHY specifications [15]. The values of power consumption for active, idle and doze states are available in [7].

\section{B. Simulation Results}

Fig. 5 analyzes the energy consumption per bit of the system with BD-PCF and PCF. The energy consumption is evaluated when stations can be in either active mode or PS mode, thus consuming energy during transmitting, receiving, listening and sleeping periods. The energy consumption of the AP, although usually considered to have unlimited energy resources, is also accounted in the experiments. When the number of stations increases, the amount of transmitted data increases proportionally. Having more data during CFP demands a higher energy consumption from mobile stations to listen to the WLAN channel during the polling process. The results reported in Fig. 5 show that the proposed BD-PCF protocol can successfully reduce the energy consumed per bit of the system, with an average reduction of $46 \%$. The largest reduction of $53 \%$ between $\mathrm{BD}-\mathrm{PCF}$ and $\mathrm{PCF}$ is recorded for the case of 50 stations.

Fig. 6 captures the throughput performance of BD-PCF with respect to PCF. BD-PCF outperforms PCF even for the scenarios with heavy traffic conditions. It shows stable improvement of around $15 \%$ in system-level throughput. The relative contribution of the AP to the total offered traffic load is also considered for throughput calculations.

Fig. 7 presents the average packet transmission delay, reported in milliseconds, measured for both BD-PCF and PCF protocols. This delay is defined as the average time elapsed since a data packet arrived at the MAC layer of the sender node and until it is successfully acknowledged by the destination. When compared to PCF, BD-PCF can reduce the transmission delay of data traffic, due to more efficient data transmission during CFPs. The difference in packet transmission delay between both curves increases with the number of stations, up to $13 \%$ for 50 stations, since more time is required for data transmission.

To further evaluate the energy saving of BD-PCF, Fig. 8 details the contribution of transmission (tx), reception (rx), idle (id) and sleep (sl) periods to the system energy consumption. In PCF (Fig. 8a), the impact of the energy consumed during

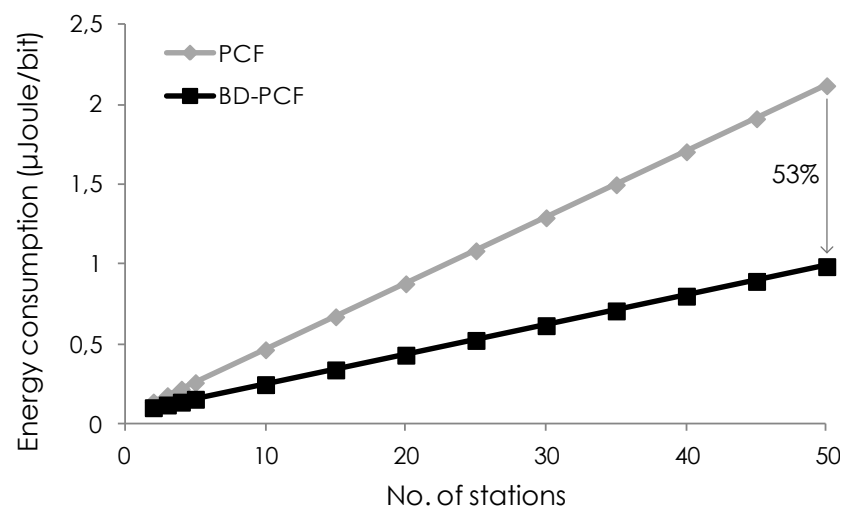

Fig. 5. System energy consumption per bit with multiple stations under heavy traffic loads.

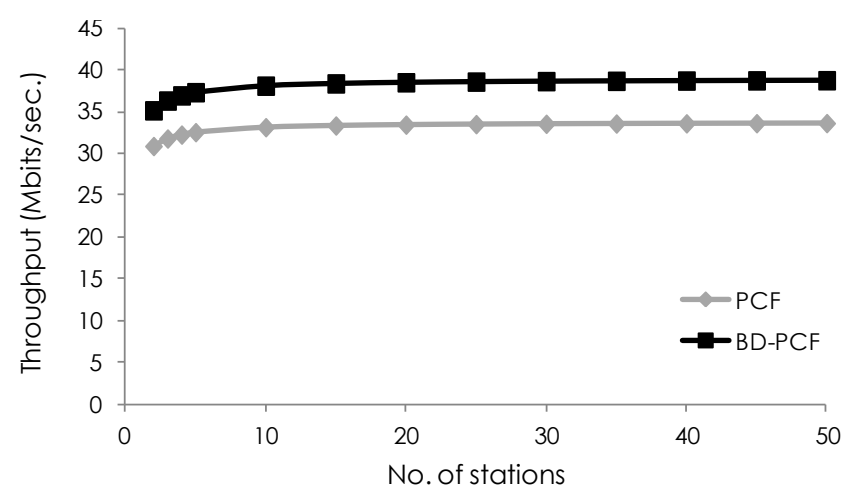

Fig. 6. System throughput with multiple stations under heavy traffic loads.

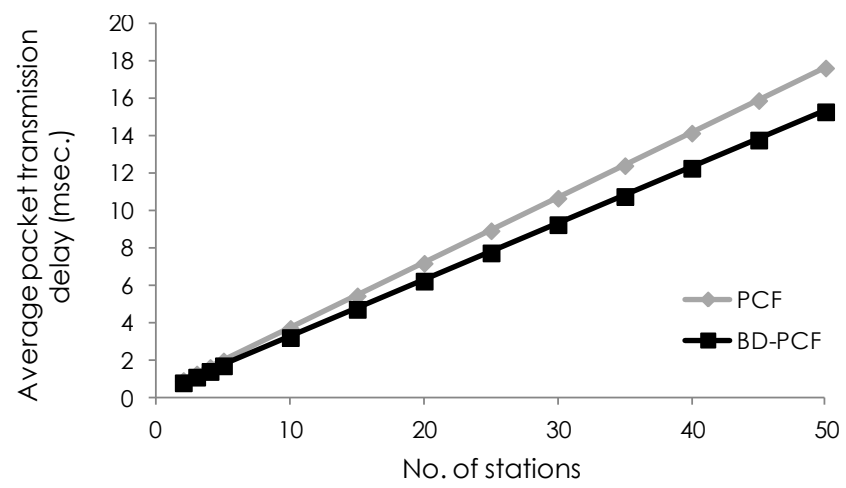

Fig. 7. Average packet transmission delay with multiple stations under heavy traffic loads.

packet reception is clearly predominant and further increases with the number of stations, reaching up to $95 \%$ of the system energy consumption for 50 stations. With this increase, the weight of the transmission energy, which is significant for few stations, becomes very small. Similarly, the idle energy, whose impact is quite low, decreases for when the number of stations is large. On the other hand, for BD-PCF the share of the total consumption that corresponds to the reception energy is lower (see Fig. 8b). This is due to the fact that under BD-PCF the stations operating progressively return to the doze state. As a result, the impact of the transmission energy becomes higher, although decreases, as well as the idle energy, with 


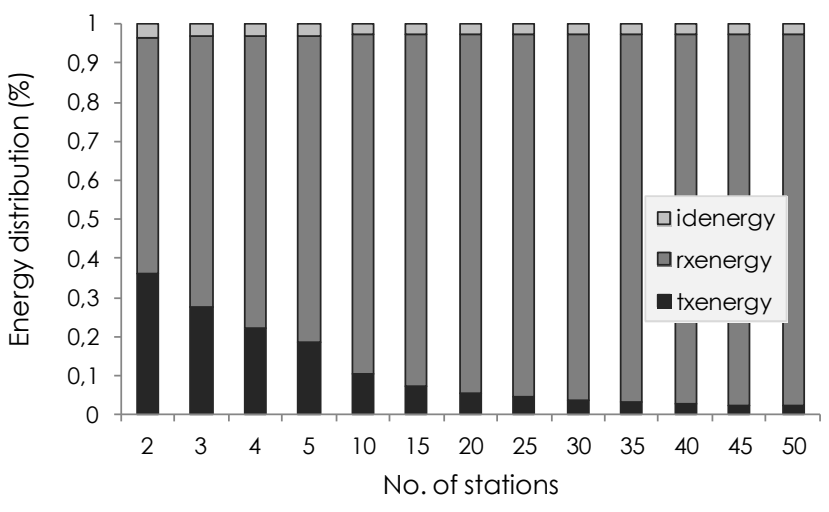

(a) PCF.

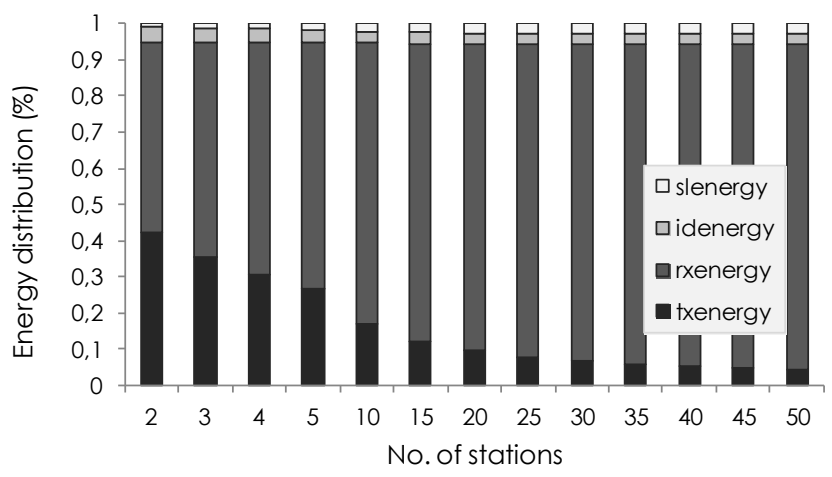

(b) BD-PCF.

Fig. 8. Distribution (\%) of system energy consumed during transmission (tx), reception (rx), idle (id) and sleep (sl) states for both BD-PCF and PCF protocols, with multiple stations under heavy traffic loads.

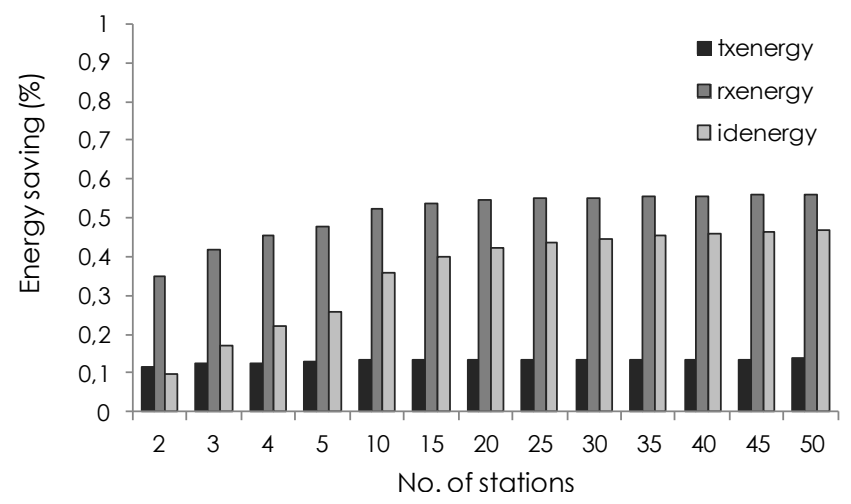

Fig. 9. System energy saving (\%) of BD-PCF against PCF during transmission (tx), reception (rx) and idle (id) periods, with multiple stations under heavy traffic loads.

the number of stations. In contrast, the weight of the sleep energy increases, with a small overall contribution due to the low energy consumed during sleep periods.

Fig. 9 shows potential gains of BD-PCF during active and idle states with respect to PCF. The maximum energy saving is achieved for packet reception. It accounts for the average reduction of $51 \%$ and a maximum of $56 \%$ for 50 stations. The idle energy is also significantly reduced, up to an energy saving gain of $47 \%$ and average of $36 \%$. For the transmission energy, a maximum gain of $13 \%$ is achieved.

\section{CONCLUSions}

This paper proposes a number of MAC enhancements for energy efficient data transmission in infrastructure WLANs. The proposed solutions improve the IEEE 802.11 PCF by granting the receiving station an opportunity to send a data packet of equal size of the received data packet to the AP. Thus, the duration of CFPs is properly estimated and disseminated through beacons by the AP. With this information, admitted stations can adopt an energy-saving strategy to stay longer in the doze state during the polling activity. Based on a simulation analysis, the results of this work prove that $\mathrm{BD}$ PCF can outperform PCF, providing a maximum gain of $15 \%$ and 53\% in terms of throughput and energy saving per bit.

A future performance evaluation of the BD-PCF protocol will consider a real hardware testbed to quantify its robustness against channel variations and its efficiency of data transmission under non-symmetric traffic flows.

\section{REFERENCES}

[1] IEEE, Part 11: Wireless LAN Medium Access Control (MAC) and Physical Layer (PHY) Specifications, IEEE 802.11 Std., 2007.

[2] S.-L. Tsao and E.-C. Cheng, "PIANO: A power saving strategy for cellular/VoWLAN dual-mode mobiles," ACM Wireless Networks, vol. 14, no. 5, pp. 683-698, 2008.

[3] J.-P. Ebert, S. Aier, G. Kofahl, A. Becker, B. Burns, and A. Wolisz, "Measurement and simulation of the energy consumption of a WLAN interface," Telecommunication Networks Group, Technische Universitt Berlin, Tech. Rep., 2002.

[4] C. E. Jones, K. M. Sivalingam, P. Agrawal, and J. C. Chen, "A survey of energy efficient network protocols for wireless networks," ACM Wireless Networks, vol. 7, no. 4, pp. 343-358, 2001.

[5] S.-L. Tsao and C.-H. Huang, "A survey of energy efficient MAC protocols for IEEE 802.11 WLAN," ACM Computer Communications, vol. 34, no. 1, pp. 54-67, 2011.

[6] A. Kpsel, J.-P. E. A. Wolisz, J. pierre Ebert, and A. Wolisz, "A performance comparison of point and distributed coordination function of an IEEE 802.11 WLAN in the presence of real-time requirements," MoMuC2000, Waseda, Tokio, Japan, pp. 1-6, Oct. 2000.

[7] Z.-T. Chou, C.-C. Hsu, and S.-N. Hsu, "UPCF: a new point coordination function with QoS and power management for multimedia over Wireless LANs," IEEE/ACM Trans. on Net., vol. 14, no. 4, pp. 807-820, 2006.

[8] R. Palacios, F. Granelli, D. Gajic, and A. Foglar, "An energy-efficient MAC protocol for infrastructure WLAN based on modified PCF/DCF access schemes using a bidirectional data packet exchange," in Proc. of IEEE CAMAD'12, Sep. 17-19, 2012, pp. 216-220.

[9] A. Kanjanavapastit and B. Landfeldt, "An analysis of a modified point coordination function in IEEE 802.11," in Proc. of the IEEE PIMRC03, vol. 2, 2003, pp. 1732-1736.

[10] C. Crespo, J.Alonso-Zrate, L. Alonso, and C. Verikoukis, "Distributed Point Coordination Function for Wireless Ad hoc Networks," in IEEE VTC Spring, 2009, pp. 1-5.

[11] J. Alonso-Zárate, C. Crespo, C. Skianis, L. Alonso, and C. Verikoukis, "Distributed point coordination function for IEEE 802.11 wireless ad hoc networks," Ad Hoc Networks, vol. 10, no. 3, pp. 536-551, 2012.

[12] A. Ganz and A. Phonphoem, "Robust SuperPoll with chaining protocol for IEEE 802.11 wireless LANs in support of multimedia applications," ACM Wireless Networks, vol. 7, no. 1, pp. 65-73, 2001.

[13] K.-C. Ting, F.-C. Kuo, B.-J. Hwang, H. Wang, and C.-C. Tseng, "A power-saving and robust point coordination function for the transmission of VoIP over 802.11," in IEEE ISPA'10, 2010, pp. 283-289.

[14] J.-R. Hsieh, T.-H. Lee, and Y.-W. Kuo, "Energy-efficient multi-polling scheme for wireless LANs," IEEE Trans. on Wireless Communications, vol. 8, no. 3, pp. 1532-1541, 2009.

[15] IEEE, Part 11: Wireless LAN Medium Access Control (MAC) and Physical-Layer (PHY) specification in the $2.4 \mathrm{GHz}$ Band, IEEE $802.11 \mathrm{~g}$ Std., 2003. 